\title{
In-Flight Total Dose Estimation Using RADEM Instrument on JUICE
}

\author{
W. Hajdas, A. Mrigakshi \\ Laboratory for Particle Physics, Paul ScherrerInstitut, CH-5232 PSI Villigen, Switzerland \\ Email: wojtek.hajdas@psi.ch
}

Received 8 December 2015; accepted 26 February 2016; published 29 February 2016

\begin{abstract}
Missions flying to giant planets frequently provide telemetry data after substantial time lag. Determination of crucial environmental characteristic sometimes detrimental for the mission health may be further delayed by duration of subsequent data analysis. We propose a fast method used in-flight to assess the electron total ionizing dose and dose rate onboard of the JUICE ESA mission to JUPITER. The procedure provides estimated values of dose rate behind various thickness of shielding using counting rates from the electron telescope EHD of the RADEM radiation hard electron monitor instrument onboard JUICE.
\end{abstract}

\section{Keywords}

Planetary Exploration, Jupiter, Radiation Environment, Detection Technology, Total Ionizing Dose

\section{Introduction}

The primary concern of the ESA JUICE mission to Jupiter is the harsh particle radiation environment [1]. Such ionizing particles passing through electronic components or materials introduce material damage caused by total dose effects, displacement damages or single events effects [2]. In extreme cases these effects can be detrimental and may even lead to device failure. Therefore, both the total ionizing dose (TID) and the displacement damage equivalent fluence (DD), must be assessed to alert spacecraft and its payload as well as to quantify radiation levels for the entire mission lifetime. In case of the JUICE mission especially electrons must be treated with extreme care as they dominate the Jovian environment both due to their very large fluxes and high energies.

In this work we present both, a concept and implementations steps for simplified method used for in-flight computation of the dose rate and total ionizing dose caused by electrons. The values are provided for a set of active volumes that are located behind layers of materials with predefined thickness. The method is based on the electron flux measurement provided by the Electron Head Detector (EHD) inside the Radiation Hard Electron Monitor (RADEM) constructed for the ESA JUICE mission. The detector is made as a telescope with eight Si-diodes used as sensors. They are enclosed inside of a shielding made of a Copper cylinder. Aluminum and Tantalum absorbers with adjusted thickness are located between the diodes making each of them sensitive to different range of energies. The method proposed in this work allows for fast determination of the electron dose rates for purpose of spacecraft alerts and payload protection. More comprehensive, detailed and more precise 
analysis of all detector systems of the RADEM instrument will be made during off-line data processing. It will provide highly accurate dose rates and total ionizing dose values for each mission segment. Such analysis is based on full spectral deconvolution and requires comparative and complementary data from all RADEM detector subsystems.

\section{Assessment of TID Using Electron Detector}

High fluxes of energetic electrons in the Jovian system are main reason for very large dose rates and total ionizing dose values reaching levels of up to $1 \mathrm{Mrad}(\mathrm{Si})$. Electrons interacting with the spacecraft components including EHD generate several radiation effects related not only to the total dose domain but also to the displacement damage one such as increase of leakage currents in diodes or operation threshold shifts in transistors. Both of them might be critical for performance of spacecraft and payload instruments. As a baseline all JUICE instruments must be qualified for high radiation dose levels. It is also valid for RADEM monitor with its components procured and qualified for the TID level of $100 \mathrm{krad}(\mathrm{Si})$. RADEM detectors however will be exposed to even higher total dose values. Based on the Jupiter JUICE mission radiation environment assessment [3] the TID values for RADEM diodes located at the top of the monitor may reach up to $300 \mathrm{krad}(\mathrm{Si})$.

Electron detector EHD is a telescope consisting of eight Si-diodes separated by absorbers. Thickness of absorbers is selected to maximize sensitivity levels of consecutive detectors to various electron energies. The values were optimized to provide eight energy bins spaced equally in a logarithmic scale and spanning over the range from $0.3 \mathrm{MeV}$ to $40 \mathrm{MeV}$. Characteristics of the EHD detector unit are given in Table 1. The EHD internal structure with a layout of Si-diodes and absorbers is showed in Figure 1. EHD will measure instantaneous spectra of electrons by detecting their energy depositions in the series of diodes of the telescope. This way, each diode of EHD will also measure the dose rate and total accumulated ionizing dose coming from electrons which directly or indirectly deposited their energy in it. The dose is a measure of energy $d E$ produced by ionizing radiation in material mass element $d M$-see (1).

Table 1. EHD Electron telescope design characteristics.

\begin{tabular}{|c|c|c|}
\hline \multicolumn{3}{|c|}{ Design Parameters } \\
\hline \multirow{2}{*}{ Cu collimator } & Height & $20.0 \mathrm{~mm}$ \\
\hline & Opening angle & $7.5^{\circ}$ \\
\hline \multirow{2}{*}{ Si diodes } & Thickness & $0.3 \mathrm{~mm}$ \\
\hline & Radius & $1.5 \mathrm{~mm}$ (top), $3.0 \mathrm{~mm}$ (rest) \\
\hline \multirow{2}{*}{ PCB } & Thickness & $0.5 \mathrm{~mm}$ \\
\hline & Radius & $11.0 \mathrm{~mm}$ \\
\hline \multicolumn{2}{|c|}{ Absorber materials } & $\mathrm{Al}$ and Ta layers \\
\hline \multicolumn{2}{|c|}{ Absorber thicknesses } & $0.4-3.8 \mathrm{~mm}$ \\
\hline \multicolumn{2}{|c|}{ Cu side/back shield thicknesses } & $5.0 \mathrm{~mm}$ \\
\hline
\end{tabular}

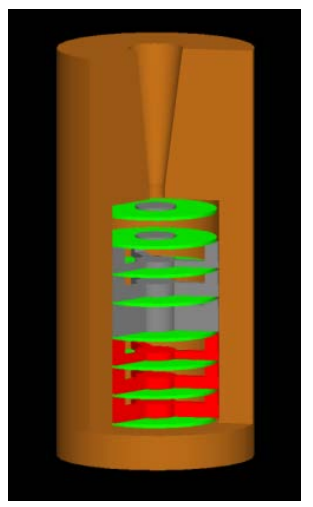

Figure 1. Cross section of the EHD telescope with set of 8 diodes and absorbers placed inside of Copper cylinder. 


$$
D=\frac{d E}{d M}
$$

EHD detects particles by recording signals from their energy depositions (above preset threshold values) in any of its diodes.

As the main function of the detector is measurement of the electron spectrum one stores coincidence patterns between the diodes. The coincidences allows for subsequent determination of the electrons spectra encountered by the JUICE satellite along its trajectory. Such spectral measurements are necessary for precise assessment of the absorbed dose or dose rates. In addition, single hit rates from each diode are stored as well. Under suitable conditions a fast estimation of dose rates may be based on single rates from individual Si-diode sensors. We present calculations of the total ionizing dose in Si-diodes using realistic spectra of the Jupiter radiation environment [3]. As the effective absorber thickness increases between each subsequent sensor, the method gives also an estimation of the dose as a function of the mean absorber thickness. The method relies on Monte Carlo simulations with GEANT4 code from CERN used to calculate energy depositions in the Si-diode sensors.

\section{TID Calculation Procedure}

Reliable computation of detector responses and accurate determination of energy depositions from incoming radiation require construction of the proper mass model. Full mass model of the detector including all its details and features of Si-diodes active volume was made using GEANT4 Monte Carlo package. Incoming electrons were generated using a GEANT4 code electron source enveloping EHD and radiating inwards in an isotropic manner. The energy spectrum of electrons was set to follow a power law distribution with an index equal to -1 . For the first series of computations simulated particles were uniformly distributed over logarithmic energy scale ranging from $0.01 \mathrm{MeV}$ to $100 \mathrm{MeV}$. This means that the same number of particles falls within any energy interval of constant width in a logarithmic energy scale. Although the EHD telescope is constructed accurately the rest of RADEM and satellite was not taken into account. Therefore the results to some extend overestimate the particle rates from electrons coming from the side. This can be regarded as the worst case dose rate estimation.

During simulation runs, energy depositions in each Si-sensor were registered and stored event by event i.e. for each generated electron which left some energy in any of eight sensors. The data were subsequently processed to create histograms with energy depositions as a function of the primary particle energy and direction.

The contribution to the dose (rate) from electrons with energy $\mathrm{E}$ and coming from direction defined by the set angles $(\Theta, \phi)$ was computed simulating their energy depositions. The total energy deposition or the dose from this direction is given by the flux convoluted with the response function and integrated over all electron energies. Subsequent integration over the whole range of incoming angles provides the total dose as is showed in (2).

$$
\frac{\Delta D}{\Delta t}=\iint d \Omega \int_{E t h r}^{\infty} d E \cdot F(E, \Theta, \phi) \cdot g(E, \Theta, \phi) \cdot \frac{\Delta E(E, \Theta, \phi)}{\Delta m}
$$

where:

$\frac{\Delta D}{\Delta t}$-dose rate

$F(E, \Theta, \phi)$-electron flux

$g(E, \Theta, \phi)$-diode response function of the EHD

$\Delta E(E, \Theta, \phi)$-energy deposition in the active volume of the EHD diode

$\Delta m$-mass of the active volume of the diode

The angular integration covers the whole solid angle while the integral over electron energies is taken from the Si-diode energy threshold. The energy deposition $\Delta \mathrm{E}$ is the full energy left in the diode by each generated electron or secondary particles such as e.g. bremsstrahlung photons produced by it. One should remark that the cosine rule for simplified calculations of energy deposition using energy loss formulas would provide only misleading if not wrong results as the electrons tends to scatter multiple times and the diode crossing direction is rather weakly related with the initial electron direction. One can therefore also assume that for each diode the mean energy deposition $\Delta \mathrm{E}$ is only weakly dependent on the initial electron energy and direction as it is later supported by the Monte Carlo simulation results. Moreover the simulations show that this energy changes very little and stays relatively constant for the whole range of the initial electron energies selected as a representative for the Jupiter environment. Having it one may replace the energy deposition in the integral by a mean energy deposition $<\Delta E>$ in 
front of it as seen in (3).

$$
\frac{\Delta D}{\Delta t}=\frac{<\Delta E>}{\Delta m} \iint d \Omega \int_{E t h r}^{\infty} d E \cdot F(E, \Theta, \phi) \cdot g(E, \Theta, \phi)
$$

Remaining part of the integral in (3) gives the particle rate $N$ in the given Si-diode for all hits above the energy threshold. This way the final formula for the dose rate will have the form given by the Equation (4).

$$
\frac{\Delta D}{\Delta t}=\frac{<\Delta E>}{\Delta m} \cdot N
$$

The formula (4) is valid for each Si-diode. One can assume that despite of relatively constant energy loss of electrons in the full range of initial energies the mean energy deposition may vary between the didoes. Thus a proper value of $\langle\Delta E>$ is calculated using Monte Carlo simulations separately for each diode. Validity of above assumptions and correctness of the derived dose rate estimation formula is provided in the next chapter.

The final in-flight computation of the dose rate using above formula is rather straightforward. The counting rates measured continuously by RADEM monitor for each of its EDH Si-diode detectors are stored in the monitor registers. The values of the active detector volume and thus its mass are provided from the on-ground calibrations. Such calibrations also give the values of mean energy depositions using realistic spectra of electrons. For the in-flight computations the values of the mean energy depositions are calculated using simulation codes using best possible spectra and mass models. Moreover the simulations can be verified and fine-tuned during on-ground calibrations. With these procedures and validated values of mean energy depositions the computation of the dose rate is based on multiplication of three numbers. As each diode provides the dose rate behind certain effective thickness, the EHD returns dose rate for the whole range of volumes located either almost on the JUICE surface or hidden deeper under a thick layer of shielding. This set of dose rates as a function of the thickness will be particularly useful for instruments with components located under different shielding layers.

\section{Simulation Results}

Monte Carlo simulations were performed with the goal to verify the main assumption behind the method for fast calculation of the electron dose. It postulates a weak dependence of the mean energy loss of the detected electron on its initial energy and initial incoming direction. Obviously for electrons coming only from the entrance opening it is fulfilled to very high degree for all SI-diodes in the telescope. It is well illustrated by Figure $\mathbf{2}$ showing experimental results from EHD Breadboard exposed to energetic electron beams at PSI within the range from 6 $\mathrm{MeV}$ to $50 \mathrm{MeV}$. The mean values of deposited energies are for both detectors very similar and independent on the electron primary energy value. An exception may be chiefly from the low energy electrons fully stopped in the Si-diode. A fraction of such events is rather small and they leave energies up to about $200 \mathrm{keV}$ in the diode. These values are very close to typical mean energy loss for electron of any energy. It is well illustrated in Figure 3 which shows energy depositions in function of the primary electron energy for all the diodes. Diode 0 is the top one with the weakest absorber and Diode 7 is the bottom on closest to the back-shield of EHD. The relatively narrow band of events up to about $10 \mathrm{MeV}$ with rough limits for energy deposition between $80 \mathrm{keV}$ and $300 \mathrm{keV}$ comes from electrons penetrating directly through the entrance window. The side shielding becomes transparent for energies near $10 \mathrm{MeV}$. Therefore the range of possible energy depositions becomes much wider. Nonetheless the most probably values are falling again between $100 \mathrm{keV}$ and $200 \mathrm{keV}$. Therefore a first observation is that independently of the electron initial energy the most probable values of deposited energies are very similar. It tends to be valid both for electrons coming directly through the entrance with a rather narrow width of possible energy loss values and for electrons coming through the shielding with much larger range of deposited energies.

Such a weak dependence of the mean deposited energy supports the presented method of the dose calculation. The second part of the assumption relates to the dependence of the mean deposited energy on the initial direction of incoming electrons. Simulation results are showed in Figure 4. Note that the direction through the entrance window corresponds to the incoming $\Theta$ angle near to $180^{\circ}$. One can see again that despite of rather variable distribution with the maximum width near the angles of about $90^{\circ}$ the mean deposited energy lies for the whole angular range between about $80 \mathrm{keV}$ and $300 \mathrm{keV}$. While more precise numerical calculations are still ongoing, one can already conclude that both for any initial energy and incoming direction the mean value of energy deposited by electron is very similar. Moreover this value is even comparable between all diodes of the EHD telescope. For example for directions within the collimator opening angle the mean values change from $130 \mathrm{keV}$ for diode 0 to 

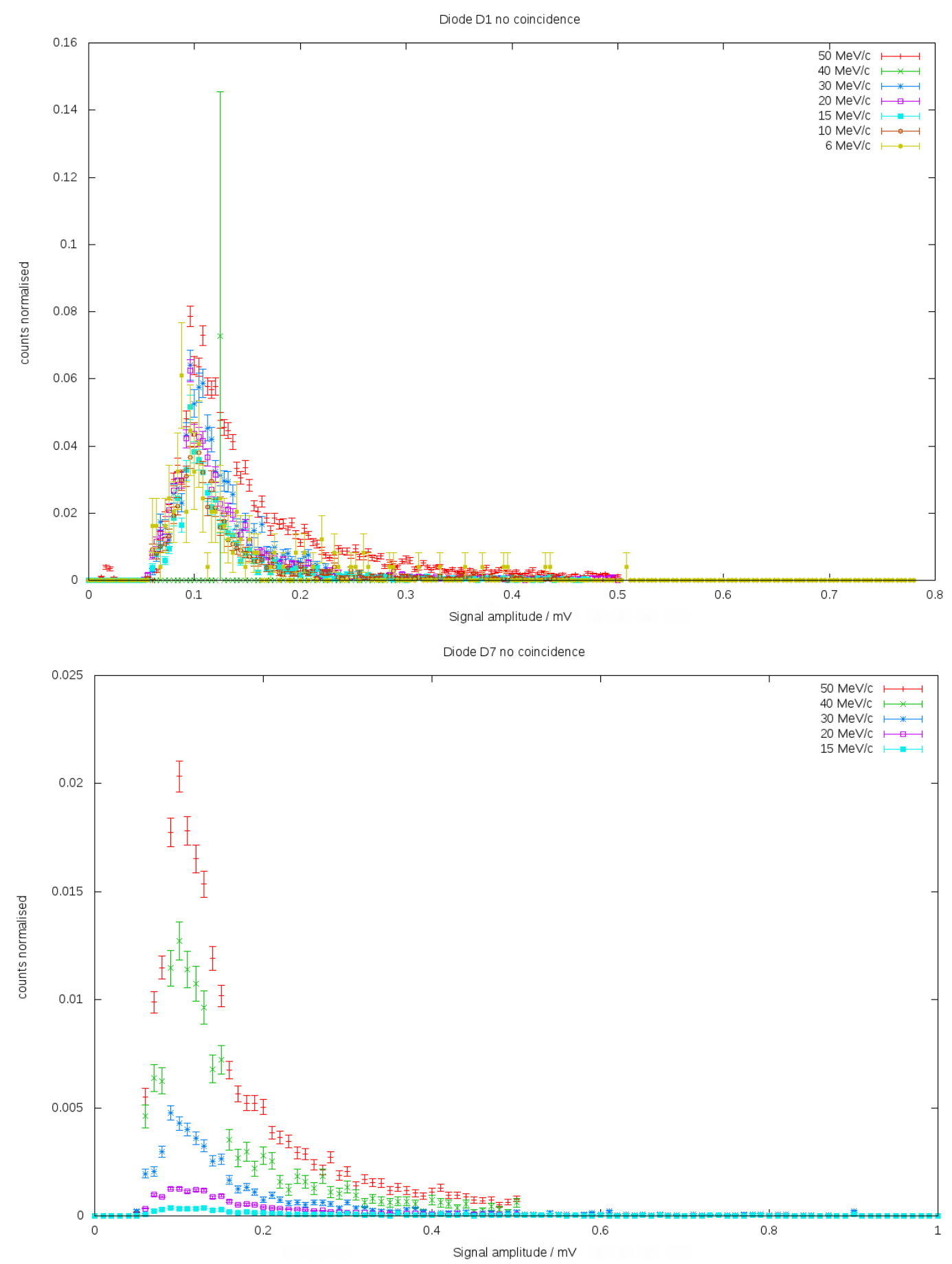

Figure 2. Mean deposited energy for diode 1 and diode 7 determined during experiments with high energy electron exposures at PSI. The EDH was exposed from the front.

$160 \mathrm{keV}$ for diode 6 . It means that the main factor contributing to the energy deposition and thus to the absorbed dose is the single counting rate observed in each particular diode.

In order to study the deposited energy further a mean value of it was computed for two cases. In the first one the electrons were coming only through the front collimator opening and in the other one the full angular range was used. The results are showed in Figure 5. The frontal events have energy depositions slightly below $150 \mathrm{keV}$ while the side events below and around $200 \mathrm{keV}$. As the side and back shielding of the EHD mass model used in 

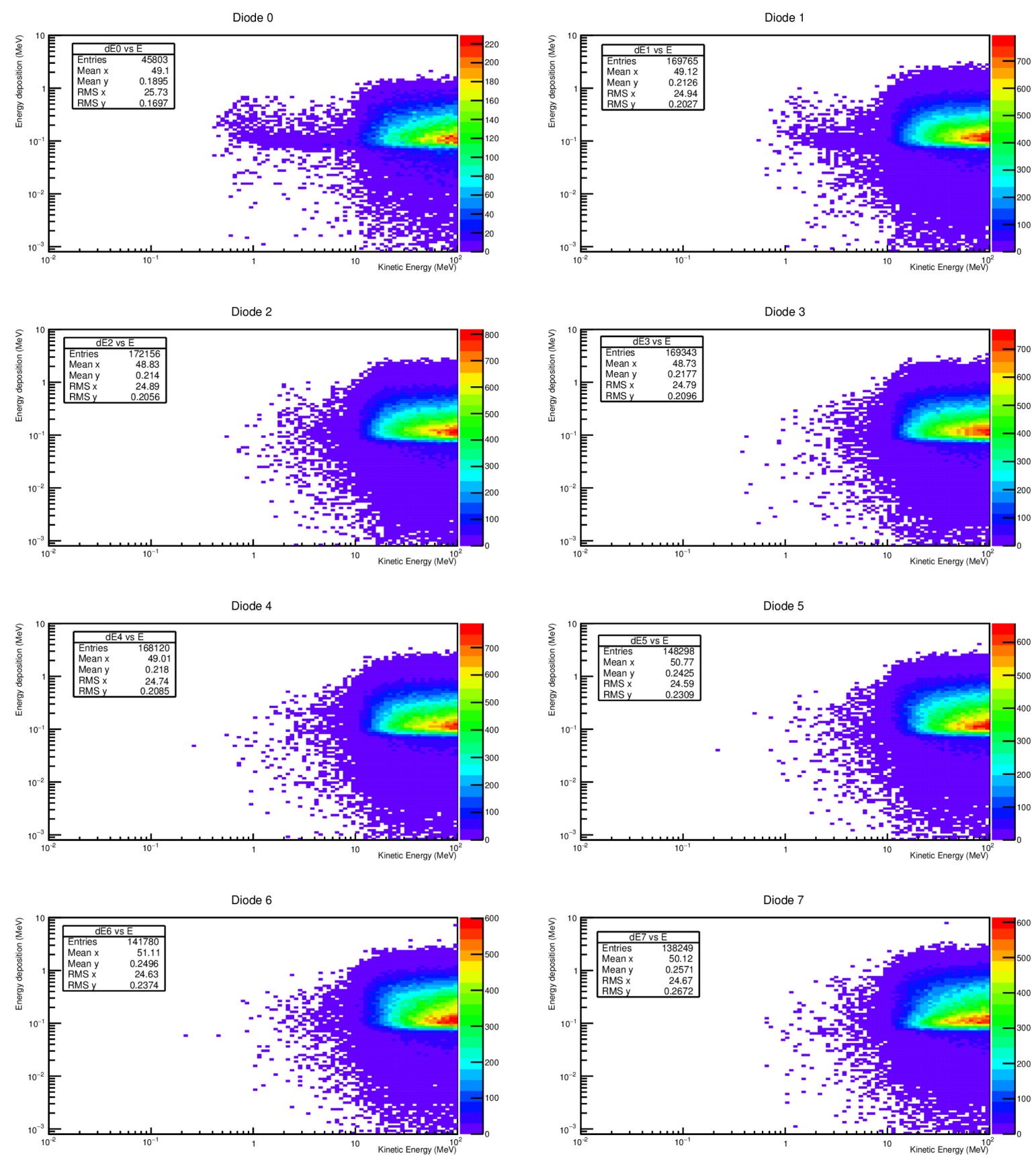

Figure 3. Energy deposition as a function of the primary electron incoming energy for all Si diodes. Results are based on full 4pi Monte Carlo simulations.

simulations is weaker than in reality due to lack for satellite and parts of RADEM monitor one can expect that the influence of side events in real case will be smaller. This would bring both mean energy depositions even closer.

Simulations presented here also support the need of having single rate counters for each diode of the EDH in RADEM. The counters are planned to be implemented for both low and high energy discriminator threshold applied to the detected signals. This will allow for further improvement of the dose calculation and minimizing systematic errors. In this implementation of the fast dose calculation the single rate counters will be used for this purpose. The counters for coincidence events will be used for spectrum unfolding and further off-line preprocessing. Precise spectrum determination will allow for accurate dose determination for any thickness of the shield. 

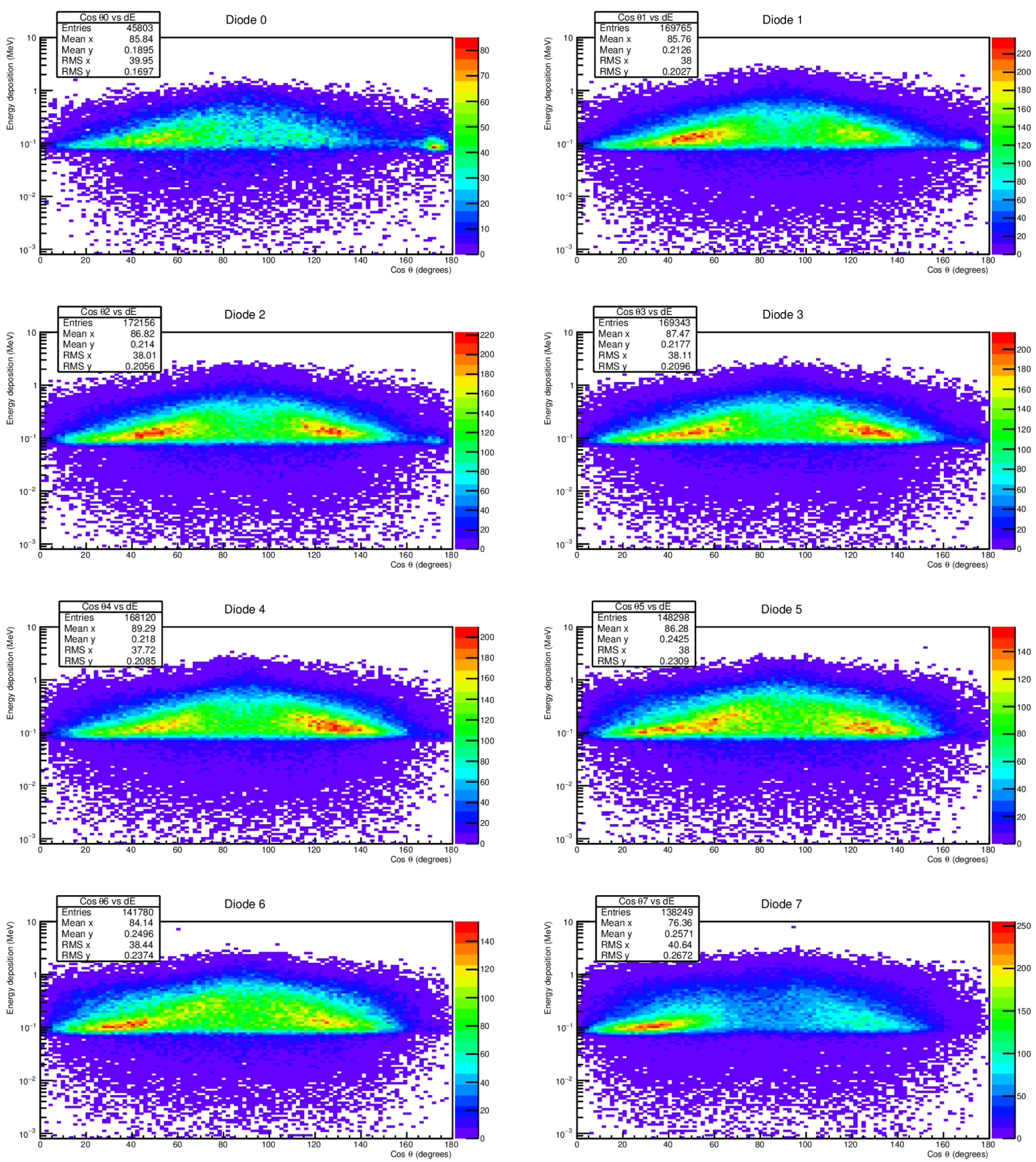

Figure 4. Energy deposition as a function of the primary electron incoming angle for all Si diodes. Results are based on full 4pi Monte Carlo simulations.

\section{Absorbed Dose}

To calculate the absorbed dose the total energy deposition that was computed for all angles and initial energies is divided by diode masses taking into account the active volumes of them as in (1). Assuming an averaged energy loss to be around $150 \mathrm{keV}$ to $200 \mathrm{keV}$ per electron the dose absorbed in the active detector volume will be 5.7E-7 rad/electron for the Diode-0. During the passage through the active radiation regions of the Jupiter belts the detector rates may reach the value of about 1E5/s. Such rates would give for Diode 0 the dose rate of about 0.056 $\mathrm{rad} / \mathrm{s}$ (or $4.9 \mathrm{krad} /$ day). Assuming slightly lower hit rates for rear diodes, it would give them correspondingly lower dose rates. Preliminary simulations showed that the rates for each next diode drop by a factor of about two 

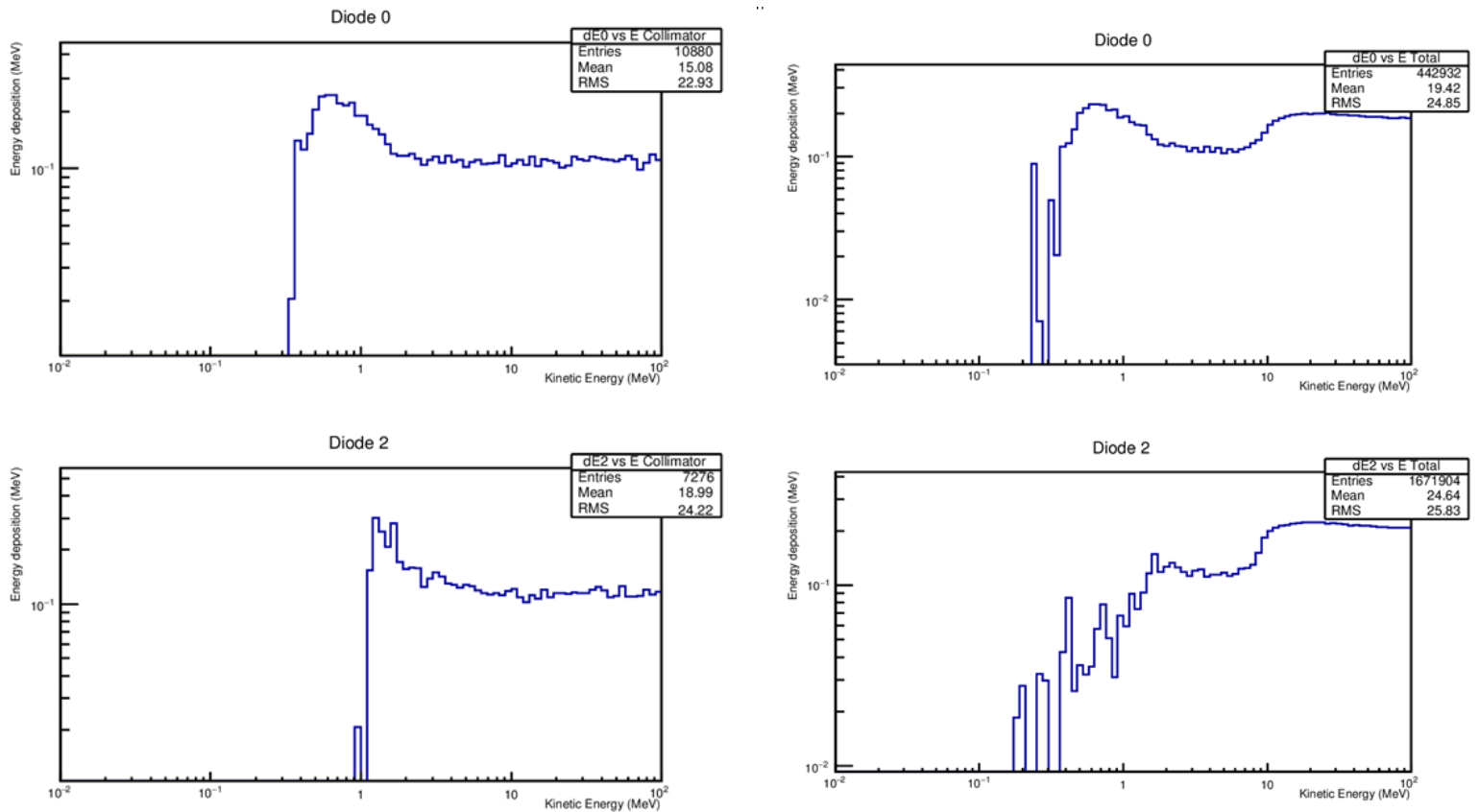

Figure 5. Mean deposited energy for diode 0 and diode 2 as a function of the primary electronenergy. Left side- for particles coming only from the front; Right side—-full 4pi simulations.

to three. This way the diode 4 has dose rates from the electrons smaller by about two orders of magnitude then diode 0 . As mentioned before, for further refinement of the absorbed dose during off-line analysis one will also take into account the total energy depositions as a function of particles type. Full spectral analysis and background subtraction will be done for each diode separately providing much more accurate dose rate assessment.

\section{Implementation}

The procedure presented in this chapter provides a technique for simple in-flight estimation of the electron induced dose rates and total absorbed dose using Si-diodes of the EHD telescope. The calculation is based on the mean energy deposition from electrons, the detector mass and the measured electron and proton counting rates. Final value of the dose rate needs these tow rates counting rate numbers from RADEM EHD as well as two fixed numbers stored in the instrument registers. Each diode of the EHD detector has two discriminators. The first one measures all counts above the low energy threshold. The second one is set higher and should count energy depositions from particles heavier than electrons. Therefore the proper electron rate is obtained by subtracting the rates measured by both thresholds as showed in the formula below.

$$
\frac{\Delta D_{i}}{\Delta t}=\frac{<\Delta E_{i}>}{\Delta m_{i}} \cdot\left(N_{i}^{\text {low }}-N_{i}^{\text {high }}\right)
$$

where:

$\frac{\Delta D_{i}}{\Delta t}$-dose rate in the diode $i$

$<\Delta E_{i}>$-mean energy deposition by electron in diode $i$

$N_{i}^{\text {low }}$-count rate above the low threshold (all particles)

$N_{i}^{\text {high }}$-count rate above the high threshold (protons and heavier particles)

$\Delta m_{i}$-mass of the active volume of the diode $i$

Based on Monte Carlo simulations and Jupiter radiation environment model, one should note that the count rate from protons is about two orders of magnitude lower than from electrons. On the other hand protons deposit few times larger energy values comparing to electrons. The mean energy deposition will be further refined by more representative Monte Carlo calculation supported by calibration measurement that includes the real detector 
thickness. It is expected that each diode will have slightly different value of $\left\langle\Delta E_{i}>\right.$. The mass of the diode is also depending on its active surface (and volume). The active detector surface will also be determined during calibration measurements. As counting rates measurements and resulting dose computation and particle separation depend on proper setting of the detector thresholds the stability and value of them are critical factors for high quality, precise measurements. The goal is to set the low energy threshold to such value that no electron signals will be cut off. The high energy threshold is to be set in such a way that the proton events are cut off in the most efficient way and without losing electron events. As we could see that the depositions from electrons can reach the values of even up to $1 \mathrm{MeV}$, the proper setting of the high threshold must be very carefully studied and precisely realized. The difficulties relate to the fact that the mean proton energy deposition may be around the value of $0.5 \mathrm{MeV}$. Thus special care must be taken in order not to cut electron events. Further steps include corrections for such factors like temperature and/or counting rate dependence of the threshold. Extensive study of all above factors will help to determine error margins for the fast dose estimation method.

\section{Summary}

A fast, straightforward procedure to estimate in-flight the electron ionizing dose and dose rate was introduced for implementation on the RADEM monitor onboard the ESA mission JUICE. It utilizes EHD electron telescope and allows for computation of the dose rate for each of its Si-diodes. The method is based on the average energy loss of electrons passing through the diodes and the single (non-coincident) counting rates. Simulation results showed that the average energy loss is only weakly dependent on the initial electron energy and also its incoming direction. The average energy loss per electron also changes weakly $(20 \%-30 \%)$ between diodes of the EHD telescope. By incorporation of the proton events collected using high level discriminators and corresponding counters the accuracy of the method can be further increased. As the mean absorber thickness of each diode varies from very thin up to relatively thick, one can provide the dose rate values behind predefined thickness of the shielding and generalize the results for the whole JUICE spacecraft. The method presented here can is to be applied for preliminary and fast dose or dose rate estimation only. More accurate computations will be based on full spectral unfolding, cross correlation between all detectors of the RADEM instrument and with final calculations supported by Monte Carlo simulations. This procedure will be only applied for off-line analysis. Above method can be further refined and validated. On the computational side more accurate simulations will be performed. They will include the full mass model of the JUICE as well as radiation environments constructed bu each mission segment. On the experimental side the method will be tested using electron beam exposures using low and high energy electron test facilities at PSI.

\section{References}

[1] Divine, N. (1974) Jupiter Radiation Belt Models. NASA Techn. Mem. 33-715, Jet Prop. Lab., Pasadena, 13 p.

[2] Johnson, R.E., Carlson, R.W., Cooper, J.F., Paranicas, C., Moore, M.H. and Wong, M.C. (2004) Radiation Effects on the Surface of the Galilean Satellites. In: Bagenal, F., Dowling, T. and McKinnon, W.B., Eds., Jupiter-The Planet, Satellites and Magnetosphere, Cambridge University, Cambridge, 485-512.

[3] Garrett, H.B., Levin, S.M. and Bolton, S.J. (2005) A Revised Model of Jupiter's Innerelectron Belts: Updating the Divine Radiation Model. Geophys. Res. Lett, 32, L04104. 